\title{
Lead-substituted barium hexaferrite for tunable terahertz optoelectronics
}

\author{
L. N. Alyabyeva (1), A. S. Prokhorov,2, D. A. Vinnik³, V. B. Anzin ${ }^{1,2}$, A. G. Ahmed ${ }^{1,4}$, A. Mikheykin, P. Bednyakov ${ }^{6}$,

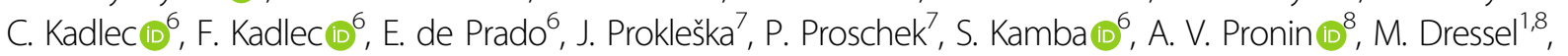 \\ V. A. Abalmasov (10), V. V. Dremov', S. Schmid ${ }^{10}$, M. Savinov' ${ }^{6}$, P. Lunkenheimer (10 ${ }^{10}$ and B. P. Gorshunov
}

\begin{abstract}
Due to their outstanding dielectric and magnetic properties, hexaferrites are attracting ever-increasing attention for developing electronic components of next-generation communication systems. The complex crystal structure of hexaferrites and the critical dependences of their electric and magnetic properties on external factors, such as magnetic/electric fields, pressure, and doping, open ample opportunities for targeted tuning of these properties when designing specific devices. Here we explored the electromagnetic properties of lead-substituted barium hexaferrite, $\mathrm{Ba}_{1-x} \mathrm{~Pb}_{x} \mathrm{Fe}_{12} \mathrm{O}_{19}$, a compound featuring an extremely rich set of physical phenomena that are inherent in the dielectric and magnetic subsystems and can have a significant effect on its electromagnetic response at terahertz frequencies. We performed the first detailed measurements of the temperature-dependent $(5-300 \mathrm{~K})$ dielectric response of single-crystalline $\mathrm{Ba}_{1-x} \mathrm{~Pb}_{x} \mathrm{Fe}_{12} \mathrm{O}_{19}$ in an extremely broad spectral range of $1 \mathrm{~Hz}-240 \mathrm{THz}$. We fully analyzed numerous phenomena with a corresponding wide distribution of specific energies that can affect the terahertz properties of the material. The most important fundamental finding is the observation of a ferroelectric-like terahertz excitation with an unusual temperature behavior of its frequency and strength. We suggest microscopic models that explain the origin of the excitation and its nonstandard temperature evolution. Several narrower terahertz excitations are associated with electronic transitions between the fine-structure components of the $\mathrm{Fe}^{2+}$ ground state. The discovered radio-frequency relaxations are attributed to the response of magnetic domains. Gigahertz resonances are presumably of magnetoelectric origin. The obtained data on diverse electromagnetic properties of $\mathrm{Ba}_{1-x} \mathrm{~Pb}_{x} \mathrm{Fe}_{12} \mathrm{O}_{19}$ compounds provide information that makes the entire class of hexaferrites attractive for manufacturing electronic devices for the terahertz range.
\end{abstract}

\section{Introduction}

The terahertz $(\mathrm{THz})$ frequency region has been the "dark horse" for the scientific community as far as technology and application are concerned. Although the theoretical predictions were rather exciting, the experimental potential did not allow to explore the proposed effects directly $^{1}$. This has changed, however, and the extensive

Correspondence: L N. Alyabyeva (aliabeva.In@mipt.ru)

${ }^{1}$ Laboratory of Terahertz Spectroscopy, Center for Photonics and 2D Materials, Moscow Institute of Physics and Technology, Dolgoprudny, Russia

${ }^{2}$ Prokhorov General Physics Institute of the Russian Academy of Sciences,

119991 Moscow, Russia

Full list of author information is available at the end of the article search for materials in recent years has begun to cover the demands of the newly developed field of $\mathrm{THz}$ electronics. Currently, the field of $\mathrm{THz}$ technologies is one of the most promising directions for the development of electronics ${ }^{2}$. The recently promoted 5G standard for broadband cellular networks operates at frequencies of tens of gigahertz. The upcoming 6G standard aims at even higher frequencies $^{3}$, and the subsequent generations will inevitably move toward the $\mathrm{THz}$ region. The application of $\mathrm{THz}$ frequencies is not limited to telecommunications, but it spans radar systems, ultrafast computer memories, nondestructive testing, safe bioscanning, security imaging, and many others ${ }^{4-6}$. The crucial need for materials with

\section{(c) The Author(s) 2021}


desired properties for particular applications has become a bottleneck for the development of $\mathrm{THz}$ technology. Since it is in the $\mathrm{THz}$ region that characteristic electronic, magnetic, and mixed-type excitations are often found, the important scientific challenge that needs to be addressed involves the search for materials whose fundamental physical properties would most adequately meet the requirements of modern technological development.

In this respect, hexaferrites recently drew considerable interest due to their distinct dielectric, magnetic, and mainly magnetoelectric properties (tunability of losses and dielectric constant, giant magnetoelasticity, multiferroicity, quantum paraelectricity) ${ }^{7-13}$ suitable for microwave and $\mathrm{THz}$ applications ${ }^{14-17}$. For example, ferromagnetic $\mathrm{BaFe}_{12} \mathrm{O}_{19}$ with an $\mathrm{M}$-type hexaferrite crystal structure (denoted as $\mathrm{BaM}$ in the following) is the most widely commercially produced magnetic material, with overall yearly market sales of billions of dollars ${ }^{18}$. The functional characteristics of substituted hexaferrites show outstanding sensitivity to doping, making these materials future candidates for novel devices of $\mathrm{THz}$ optoelectronics. Doped M-type hexaferrites exhibit superior magnetic characteristics, including the ferromagnetic resonance (FMR) frequency located in the $\mathrm{THz}$ range, record values of the coercive force of up to $40 \mathrm{kOe}^{19}$, and formation of a new type of Jahn-Teller sublattice ${ }^{20}$. Doping BaM with lead is expected to systematically change its dielectric properties. In fact, BaM is relatively transparent in the $\mathrm{THz}$ range ${ }^{21}$, whereas lead ferrite $\mathrm{PbFe}_{12} \mathrm{O}_{19}$ effectively shields $\mathrm{THz}$ radiation ${ }^{22}$. Despite constituting a core material for the magnetic device market, hexaferrites are rather poorly studied from the dielectric standpoint. In other words, the practical development of this promising class of materials calls for thorough studies of their fundamental physical properties. In particular, this concerns fundamental processes that determine the electromagnetic properties of hexaferrites at $\mathrm{THz}$ frequencies. However, data on their electromagnetic responses, including those in the $\mathrm{THz}$ range, are lacking to date.

To fill this gap, we report here the characterization of the electromagnetic properties in the $\mathrm{Hz}$ to $\mathrm{THz}$ frequency range of one of the most well-known representatives of the hexaferrite family, lead-substituted barium hexaferrite $\mathrm{Ba}_{1-x} \mathrm{~Pb}_{x} \mathrm{Fe}_{12} \mathrm{O}_{19}(x=0.1,0.2)$. For the studies, we managed to synthesize a series of unique highquality single crystals of the compounds; synthesis, characterization, and sample preparation procedures are described in detail in Supplemental Information (Chapter SI1). We found that their $\mathrm{THz}$ dielectric responses include strong soft excitations that can be easily tuned by temperature variation. The discovered $\mathrm{THz}$ resonance (soft mode) exhibits a highly unusual temperature behavior never detected in hexaferrites or other incipient ferroelectrics. We propose approaches that explain the nonclassical temperature dynamics of this "soft mode" on a microscopic level. Moreover, in the $\mathrm{Hz}-\mathrm{MHz}$ region, several temperature-activated relaxation processes were detected. In addition, we observed signs of an almost temperature-independent narrow resonance in the gigahertz region. Together with infrared (IR) lattice vibrations, the observed broadband electromagnetic response in the studied hexaferrites spans over 14 decades in frequency, and it involves processes of different natures; this enables us to draw a global picture of the microscopic mechanisms responsible for the observed phenomena. In this work, we demonstrate that admixing $\mathrm{Pb}$ dramatically alters the $\mathrm{THz}$ response of the BaM. The possibility of effective targeted tuning of the functional characteristics of substituted hexaferrites by doping significantly extends the area of their applications in $\mathrm{THz}$ electronics.

\section{Results and discussion}

In the following, we first focus on a detailed consideration of the spectra and spectral features that we observed at different frequencies and at different temperatures, offer an interpretation of the phenomena that are the origin of these features, and in the concluding section, we consider our results in the context of the possibility of their practical use.

\section{THz spectra, the soft mode}

The most intriguing fundamental result of the present study is the observation of an excitation at $\mathrm{THz}$ frequencies whose frequency position and dielectric strength reveal strong and highly unusual dependences on the temperature, as seen in Fig. 1 in the $\mathrm{THz}\left(5-70 \mathrm{~cm}^{-1}\right)$ spectra of real $\varepsilon^{\prime}(v)$ and imaginary $\varepsilon^{\prime \prime}(v)$ permittivity. For the experimental details, see the "Methods" section. The growth and characterization of the crystals are presented in chapter SI1 (see also Figs. SI1-SI5 and Tables 1 and 2 therein) of the Supplementary Information (SI). The crystal structure is described in chapter SI2 of the SI and presented in Fig. SI2-1.

Figure 2a, b demonstrates that, in the $x=0.1$ compound, a drastic change in the resonance frequency position of the soft mode occurs (Fig. 2a), which strongly contrasts with the temperature-independent behavior of the position of the lowest-frequency optical phonon at $92 \mathrm{~cm}^{-1}$ (Fig. 2b and Fig. SI2-2 in Supplementary Information). Before discussing the behavior and the origin of the discovered soft excitation in more detail, we comment on four narrower absorption lines that are resolved at the lowest temperatures on the high-frequency wing of the excitation; they are marked by arrows in Fig. 1b, c. Comparing the frequency positions, intensities, and temperature evolution of these lines with those seen in the $\mathrm{THz}$ spectra of isostructural compounds, i.e., in $\mathrm{Ba}_{1-x} \mathrm{~Pb}_{x} \mathrm{Fe}_{12} \mathrm{O}_{19}$ with 


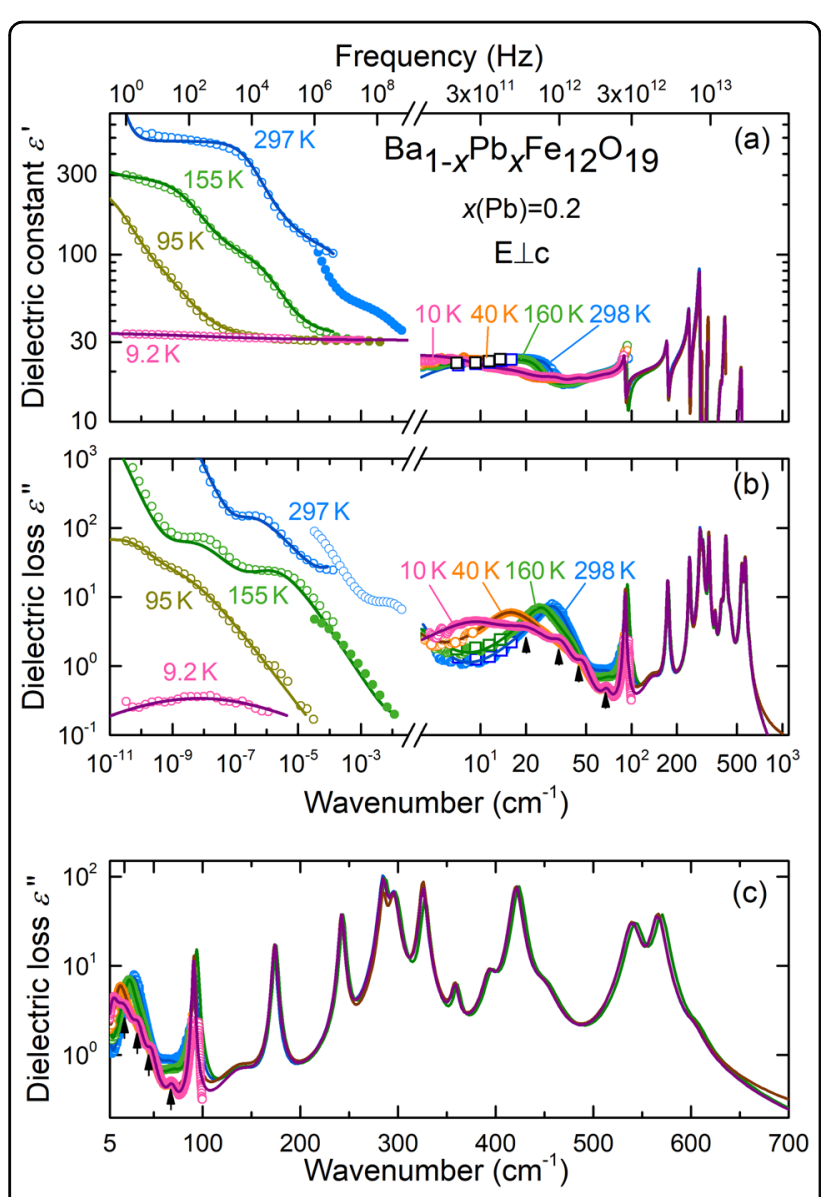

Fig. 1 Broadband (RF-THz-IR) spectra of the complex dielectric permittivity of $\mathrm{Ba}_{1-x} \mathrm{~Pb}_{x} \mathrm{Fe}_{12} \mathrm{O}_{19}, x=0.2$, measured at selected temperatures for polarization $E \perp c$. Panel (a) represents real part of the permittivitty, panels $(\mathbf{b}, \mathbf{c})$ illustrates logarithmic and linear scaled spectra of imaginary part of the permittivity. Dots correspond to experimental data; lines correspond to results of model processing using Eqs. (1) and (4) for resonances and overdamped absorptions, respectively. Open squares represent the $\mathrm{THz}$ data obtained at frequencies of interference maxima in the $\mathrm{THz}$ transmission coefficient of the plane-parallel sample, providing an enhanced accuracy in the determination of the sample's dielectric parameters ${ }^{80}$. Four relatively weak absorption lines resolved at $10 \mathrm{~K}$ (shown by arrows in $\mathbf{b}, \mathbf{c}$ ) correspond to electronic transitions of $\mathrm{Fe}^{2+}$, also known from other $\mathrm{Pb}$-substituted compound $\mathrm{BaFe}_{12} \mathrm{O}_{19}{ }^{23-25,81}$. The broad $\mathrm{THz}$

absorption band reveals a dramatic frequency softening upon cooling, as it shifts from $\approx 30 \mathrm{~cm}^{-1}$ at $298 \mathrm{~K}$ to $\approx 10 \mathrm{~cm}^{-1}$ at $10 \mathrm{~K}$. The symbols below $10^{-2} \mathrm{~cm}^{-1}$ represent the results from dielectric spectroscopy at $1 \mathrm{~Hz}-300 \mathrm{MHz}$ (open circles: from the frequency-response analysis; closed circles: from the reflectometric coaxial technique). The marked frequency-temperature-dependent response observed at these frequencies is discussed in the text. Note the changes of scale at the frequency axes. c shows linear scaled THz-IR spectra.

$x=0.7^{23}, x=0.6$ and $0.8^{24,25}$, we conclude that the four resonances observed in $\mathrm{Ba}_{1-x} \mathrm{~Pb}_{x} \mathrm{Fe}_{12} \mathrm{O}_{19}$ with $x=0.1$ $(x=0.2)$ at $64(68), 47(48), 34(34), 22(21) \mathrm{cm}^{-1}$ have the same origin. We associate these resonances with electronic transitions between the fine-structure components of the
${ }^{5} \mathrm{E}$ ground state of tetrahedrally coordinated $\mathrm{Fe}^{2+}$. The presence of $\mathrm{Fe}^{2+}$ ions in $\mathrm{BaM}$ and in $\mathrm{Pb}$-substituted $\mathrm{BaM}$ is due to both growth defects (mainly oxygen vacancies) and sharing the outer $6 \mathrm{~s}^{2}$ electron pair of $\mathrm{Pb}^{2+}$ with the $\mathrm{Fe}^{3+}$ ion. A similar effect was observed in $\mathrm{Ti}^{4+}$-substituted hexaferrites ${ }^{26}$, where $\mathrm{Fe}^{2+}$ ions maintain the charge neutrality of the compound with aliovalent doping. In addition, in Ti-substituted $\mathrm{BaM}\left(\mathrm{BaTi}_{0.75} \mathrm{Fe}_{11.25} \mathrm{O}_{19}\right)^{20}$, calculations of the adiabatic potential showed that, for $\mathrm{Fe}^{2+}$ in the BaM matrix, tetrahedral coordination was the most energetically favorable. We note that the intensities of the lines are higher at $x=0.2 \mathrm{Ba}_{1-x} \mathrm{~Pb}_{x} \mathrm{Fe}_{12} \mathrm{O}_{19}$ compound compared to the compound with $x=0.1$, which is in agreement with our assumption on the nature of these absorption lines due to the presence of the $\mathrm{Pb}^{2+}$ ions.

To determine whether some magnetic excitation can be the origin of the discovered $\mathrm{THz}$ soft mode, similar to $Y$ and $Z$-type hexaferrites ${ }^{27,28}$, we performed $\mathrm{THz}$ spectral measurements in external magnetic fields of up to $7 \mathrm{~T}$ (Faraday geometry) at temperatures of $4-300 \mathrm{~K}$. No influence of the magnetic field on the spectra was detected, indicating a purely dielectric nature of the soft excitations in both $x=0.1$ and $x=0.2$ compounds. An additional confirmation of their dielectric origin is provided by the shape of the reflection coefficient spectrum, as demonstrated and discussed in Supplementary Information, Chapter SI3 and illustrated in Fig. SI3-1. This justifies the use of the dielectric Lorentzian expression (1) for least-square processing of the THz spectra $\varepsilon^{\prime}(v)$ and $\varepsilon^{\prime \prime}$ (v) to obtain parameters of the soft modes observed in both

$$
\begin{aligned}
& \mathrm{Ba}_{1-x} \mathrm{~Pb}_{x} \mathrm{Fe}_{12} \mathrm{O}_{19} \text { samples, } x=0.1 \text { and } x=0.2 \text { : } \\
& \varepsilon^{*}(\nu)=\varepsilon^{\prime}(\nu)+i \varepsilon^{\prime \prime}(\nu)=\sum_{j} \frac{f_{j}}{\nu_{j}^{2}-\nu^{2}+i \nu \gamma_{j}}+\varepsilon_{\infty}
\end{aligned}
$$

In Eq. (1), $f_{j}=\Delta \varepsilon_{j} v_{j}^{2}$ is the oscillator strength of the $j$ th resonance, $\Delta \varepsilon_{j}$ is its dielectric strength, $v_{j}$ represents the resonance frequency, $\gamma_{j}$ is the damping factor, and $\varepsilon_{\infty}$ is the high-frequency dielectric constant. We used the high-frequency dispersion-free parts of IR reflectivity spectra (Fig. SI2-2) to determine the $\varepsilon_{\infty}$ values: $\varepsilon_{\infty}=7.06$ for $x=0.1$ and $\varepsilon_{\infty}=6.92$ for $x=0.2$. The temperature dependences of the $\mathrm{THz}$ soft mode parameters (dielectric strength $\Delta \varepsilon$, peak frequency $v$, damping $\gamma$, and oscillator strength $\Delta \varepsilon v^{2}$ ) for both compounds are presented in Fig. 2c-f). Note that the modes are not of relaxational but of resonance type, which is evidenced by the dispersion in the real permittivity $\varepsilon^{\prime}(v)$, typical of underdamped excitations, and by the small values of relative damping, $\gamma / v<1$. The frequency positions of the modes in both compounds nearly coincide, and they reveal significant decreases 


$$
\mathrm{Ba}_{1-x} \mathrm{~Pb}_{x} \mathrm{Fe}_{12} \mathrm{O}_{19}, \mathrm{E} \perp \mathrm{C}
$$
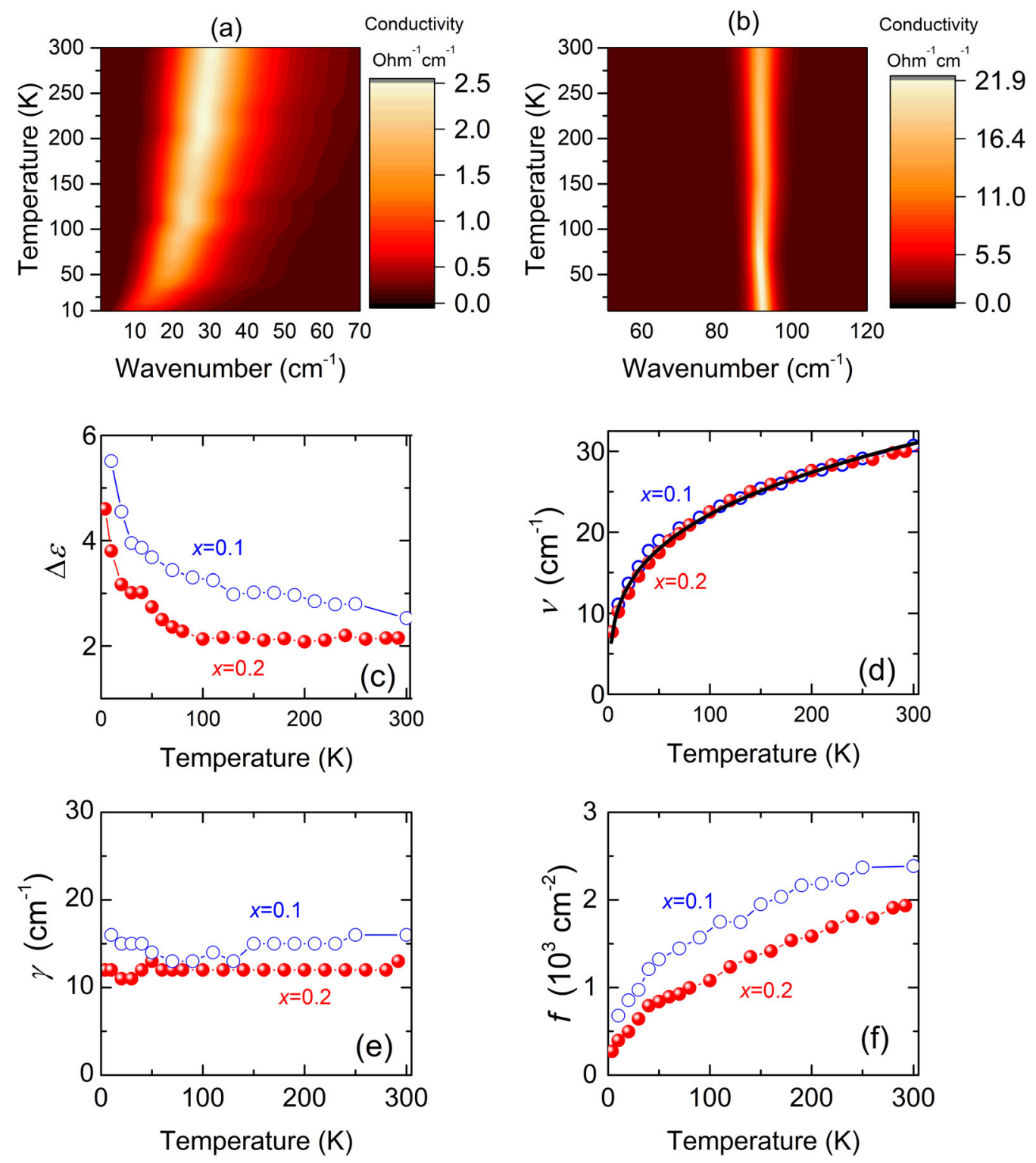

Fig. 2 The THz soft mode. 2D plots of temperature-dependent spectra of the real part of AC conductivity ( $\left.\sim \varepsilon^{\prime \prime}\right)$ of the THz soft excitation (a) and the lowest $\mathrm{E}_{1 \mathrm{u}}$ optical phonon (b) for the $\mathrm{Ba}_{1-x} \mathrm{~Pb}_{x} \mathrm{Fe}_{12} \mathrm{O}_{19} \mathrm{crystal}, x=0.1$. c-f: parameters of the soft $\mathrm{THz}$ excitation as a function of temperature for $\mathrm{Ba}_{1-x} \mathrm{~Pb}_{x} \mathrm{Fe}_{12} \mathrm{O}_{19}, x=0.1$ and $x=0.2$ crystals: dielectric strength (c), resonance frequency (d), damping factor (e), and oscillator strength (f). The black line in $\mathbf{d}$ shows a fit of the resonance frequency for $x=0.1$ with the power-law dependence $\nu^{2}=A\left(T-T_{C}\right)^{\delta}$, yielding $\delta=0.5-0.6, T_{c} \approx 3.3 \mathrm{~K}$.

upon cooling down, from $\approx 30 \mathrm{~cm}^{-1}$ at room temperature to $\approx 10 \mathrm{~cm}^{-1}$ at $10 \mathrm{~K}$ (see Fig. $2 \mathrm{~d}$ ). This softening of the frequency is accompanied by a strong increase in the dielectric strengths $\Delta \varepsilon$ that differ slightly in the two materials.

The dependences of the resonance frequency positions $v(T)$ and dielectric strengths $\Delta \varepsilon(T)$ of the excitations resemble the temperature behavior of ferroelectric soft modes originating in structural phase transitions in ferroelectric materials ${ }^{29,30}$. However, in the present case, the $v(T)$ and $\Delta \varepsilon(T)$ temperature evolutions do not obey the corresponding Cochran, $\nu^{2}=A\left(T-T_{\mathrm{C}}\right), \quad$ and Curie-Weiss, $\Delta \varepsilon=C\left(T-T_{\mathrm{C}}\right)^{-1}$, laws $\left(T_{\mathrm{C}}-\right.$ Curie temperature, $A-$ Cochran constant, $C$-Curie constant) typical of ferroelectrics. Instead, the temperature behavior of the resonance frequencies clearly follows power-law 
dependences

$$
\nu^{2}=A\left(T-T_{\mathrm{c}}\right)^{\delta}
$$

with the best fit parameters $\delta=0.5-0.6$ and critical temperatures $T_{\mathrm{c}} \approx 3.3 \mathrm{~K}$ for $x=0.1$ and $T_{\mathrm{c}} \approx 1.5 \mathrm{~K}$ for $x=$ 0.2 compounds. In addition, the damping constants remain, within the experimental uncertainty, temperature independent in the entire temperature range; this is also unusual for "standard" ferroelectric soft modes. One more specific feature of the soft excitations observed in the $\mathrm{Ba}_{1-x} \mathrm{~Pb}_{x} \mathrm{Fe}_{12} \mathrm{O}_{19}$ compounds should be pointed out: their oscillator strengths strongly decrease as the temperature is lowered (Fig. 2f), which is in strong contrast to regular ferroelectrics, wherein the soft modes usually maintain their oscillator strengths constant with temperature. Such a weakening could occur if the excitation was coupled to an additional vibrational process. Since no noticeable change with temperature in the total dielectric contributions of all IR-active phonons (Fig. 1c) was detected, the strengths of the soft modes might be transferred to the lower-frequency gigahertz resonance seen in Fig. SI4-1 or to acoustical phonons. Unfortunately, parameters of the gigahertz resonance could only be determined from the data extrapolated from $\mathrm{THz}$ and radiofrequency ranges (see below), and consequently, its oscillator strength is found with a rather large uncertainty. Nevertheless, it is clearly seen from Fig. SI4-2 (panel d) that the excitation strength does not show any sign of increase upon cooling. Therefore, we exclude its coupling to the soft mode. Further detailed microwave dielectric measurements are required to gain additional information on this resonance. Possible coupling of the mode to acoustic phonons is discussed below.

We note that the discovered soft excitation is observed for polarization $E \perp c$, i.e., when the electric field component of the probing $\mathrm{THz}$ radiation couples to the electric dipole moments that exist within the $a b$-plane. Previous studies on hexaferrites revealed temperature-dependent excitations in another geometry, $E|| c$. For example, in ref. ${ }^{21}$, a soft excitation (though at slightly higher frequencies) and the corresponding incipient ferroelectric temperature behavior of the low-frequency dielectric permittivity were observed in undoped $\mathrm{BaM}$ in the $E|| c$ response, with no indications of any soft mode-like effects in the orthogonal polarization $E \perp c$, as in the present case.

We suggest the following two mechanisms that can be responsible for the appearance of the unusual soft mode in $\mathrm{Pb}$-doped hexaferrites.

In the hexaferrite lattice, the $\mathrm{Ba}$ cuboctahedron is symmetrically surrounded by three bipyramids. The Ba ions occupy central positions in the oxygen cuboctahedron with six oxygen ions surrounding each $\mathrm{Ba}$ ion in the $a b$-plane (Fig. SI2-1b, top). When Ba is substituted with
$\mathrm{Pb}$, the interaction of the lone $6 \mathrm{~s}^{2}$ electron pair of $\mathrm{Pb}^{2+}$ with neighboring oxygens leads to the shortening of $\mathrm{Pb}-\mathrm{O}$ bonds $^{22}$ in comparison to $\mathrm{Ba}-\mathrm{O}$ bonds ${ }^{31}$, which manifests itself in an off-center shift of $\mathrm{Pb}^{2+}$ toward one of the oxygen vertices $^{22}$ (Fig. SI2-1b, bottom). This, in turn, leads to a distortion of the ionic surroundings, redistribution of electron density, and the appearance of a net electric dipole moment in the $a b$-plane. (If the $\mathrm{Pb}$ ions were located in the center of its oxygen coordination, the dipole moments induced in the three surrounding trigonal bipyramids would compensate for each other, and the net dipole moment would be zero). Since the $\mathrm{Pb}$ ion is surrounded by six oxygen atoms, at low enough temperature, it can find itself in one of six local minima within the $a b$-plane.

At sufficiently high temperatures, the $\mathrm{Pb}$ ion will be able to hop between the six potential wells, which can be effectively regarded as if the corresponding induced dipole moment changes its direction during the whole 360 degree rotation within the $a b$-plane. Due to the longrange character of the electric dipole-dipole interaction, separate dipoles of the described type will interact, with the tendency toward ordering at certain low temperatures, as happens in paraelectric phases of ferroelectrics. One can expect that the dielectric response of such coupled dipolar systems can be expressed in the form of soft excitation analogous to the paraelectric soft modes in systems with a crossover from the displacive to the order-disorder type of ferroelectric phase transition (see, e.g., refs. ${ }^{29,30}$ ). When the temperature decreases and the thermal energy $k_{\mathrm{B}} T$ becomes smaller than the energy barrier $A$ between the potential wells (here $k_{\mathrm{B}}$ is the Boltzmann constant), a certain fraction of the $\mathrm{Pb}$ ions progressively resides in the wells, and the fraction of quasi-free dipoles decreases in accordance with the Boltzmann exponential factor $\exp \left(-A / k_{\mathrm{B}} T\right)$. The dielectric response of such a system of coupled rotating electric dipoles was analyzed within the mean-field approach in refs. ${ }^{32,33}$ for the rotational potential felt by a dipole containing two and four potential minima, respectively. According to ref. ${ }^{32}$, for the case of a potential with two wells, at high temperatures, when the thermal energy exceeds the barrier height $A$ separating the wells $\left(k_{\mathrm{B}} T>\right.$ $A$ ), there will be an optical soft mode in the dielectric spectra that originates in freely interacting rotating dipoles. During cooling, the number of these dipoles that are responsible for the soft mode-like response will decrease proportionally to the Boltzmann exponent exp $\left(-A / k_{\mathrm{B}} T\right)$, and correspondingly, progressively more dipoles will be localized within the wells and respond via librations (restricted rotations) to external perturbations. Depending on the coupling strength and barrier height, the soft mode dielectric strength $\Delta \varepsilon$ (its contribution to the static permittivity) can follow the Curie-Weiss 
dependence $\Delta \varepsilon=C\left(T-T_{\mathrm{C}}\right)^{\gamma}, \gamma=-1$, or exhibit certain deviations from it toward lower absolute values of the exponent, $|\gamma|<1$. Along with that, the temperature dependence of the soft mode frequency is also expected to reveal deviations from the Cochran law $\nu^{2}=A\left(T-T_{\mathrm{C}}\right)$, as demonstrated in ref. ${ }^{34}$ for the case of interacting classical anharmonic oscillators in the two-well potential. Since the abovementioned mean-field results predict the existence of the two principal features we observe in our experiments-the soft mode and the decrease in its strength upon cooling-we suggest that the described considerations can be qualitatively applied to our case when the rotating dipoles are produced by $\mathrm{Pb}$ ions experiencing six-well potential. It is worth noting that the described model was effectively used to interpret the paraelectric response of isolated nanoconfined polar water molecules embedded in beryl crystals ${ }^{35}$.

As an alternative explanation, the peculiar dependence of the THz soft mode on temperature, $\nu \propto\left(T-T_{\mathrm{c}}\right)^{\delta}$ with $\delta \approx 0.25$, observed in our experiment can be explained by ion oscillations in the quartic potential of the form $V(Q)=(a / 2) Q^{2}+(b / 4) Q^{4}$ (here $a$ and $b$ are constants, $Q$ is a coordinate). Indeed, for large values of the coordinate $Q$, a thermally averaged harmonic part of the potential $V(Q+\Delta Q)$ as a function of the fluctuation $\Delta Q$ can be written as $V_{\mathrm{h}}(\Delta Q)=\left(a+3 b Q^{2}\right) \Delta Q^{2} / 2$. For small values of the coefficient $a, a \approx 0$, which can be expected for small values of $T_{\mathrm{c}}$, the thermal average of the coordinate in the classical limit (at high temperatures) is given by the equipartition theorem as $b Q^{4}=k_{\mathrm{B}} T$. Thus, we can estimate $Q^{2}=\left(k_{\mathrm{B}} T / 3 b\right)^{1 / 2}$ (where we have assumed $Q^{4}=3 Q^{22}$ as for the normal distribution), and we obtain a temperature dependence of the soft mode frequency, $\omega^{2} \approx\left(3 b k_{\mathrm{B}} T\right)^{1 / 2} / m$, close to that observed experimentally. According to recent X-ray diffraction data ${ }^{36}$, only $\mathrm{Pb}$ and $\mathrm{Fe}(2)$ ions have large mean square displacements in the $a b$-plane, which may indicate anharmonicity of their potentials: for $x=0.23,0.44$ and 0.8 , the square displacements are $0.0252,0.0436$, and $0.0687 \AA^{2}$ for the $\mathrm{Ba} /$ $\mathrm{Pb}$ ions and 0.0097, 0.0114, and $0.0155 \AA^{2}$ for the $\mathrm{Fe}(2)$ ions, respectively. We assume that the largest displacements correspond to the $\mathrm{Pb}$ - and $\mathrm{Fe}$-ion potentials, while the smaller displacements are due to the presence of $\mathrm{Ba}$ ions and statistical averaging. Given the measured phonon frequency of approximately $\omega=2 \pi \cdot 30 \mathrm{~cm}^{-1}$ at $T=300$ $\mathrm{K}$, we estimate $b=8054 \mathrm{meV}^{-4}$ for $\mathrm{Pb}$ ions and $b=584 \mathrm{meV}^{-4}$ for $\mathrm{Fe}(2)$ ions. The calculated ground state square displacement of $0.0219 \AA^{2}$ for the $\mathrm{Fe}(2)$ ion in the potential with the above coefficients $b$ and $a=0$ is consistent with the experimental data. At the same time, the calculated square displacement for the $\mathrm{Pb}$ ion, 0.0059 $\AA^{2}$, turns out to be significantly smaller. Thus, we conclude that the observed soft mode may be due to the anharmonic softening of $\mathrm{Fe}(2)$ ion oscillations. These ions lie in the same plane as $\mathrm{Ba} / \mathrm{Pb}$ ions and can be affected by the nearest $\mathrm{Ba} / \mathrm{Pb}$ substitution.

With the energy spectrum $E_{n}$ and wave functions of the $\mathrm{Fe}(2)$ ions in the quartic potential, we calculate the corresponding imaginary part of the dielectric susceptibility ${ }^{37}$

$$
\alpha^{\prime \prime}=\frac{1}{\hbar Z} \sum\left(e^{-E_{n} / k_{\mathrm{B}} T}-e^{-E_{m} / k_{\mathrm{B}} T}\right) \frac{\left|x_{n m}\right|^{2} \gamma_{n m}}{\left(\nu+\nu_{n m}\right)^{2}+\gamma_{n m}^{2}},
$$

where $Z=\sum e^{-E_{n} / k_{\mathrm{B}} T}$ is the partition function, $\nu_{n m}=\left(E_{n}-E_{m}\right) / \hbar$ is the transition frequency between states $n$ and $m, x_{n m}$ is the matrix element of the coordinate along the electric field (which we suppose coincides with the coordinate $Q$ ), and $\gamma_{n m}$ is the sum of the widths of the two states. The calculated real part of the alternating current (AC) conductivity, $\sigma^{\prime}=\nu \alpha^{\prime \prime}$, for $\gamma_{n m}=10 \mathrm{~cm}^{-1}$, is shown in Figure SI5-1 (AC conductivity characterizes electromagnetic absorption due to ionic oscillation). The calculated peak conductivity (which is related to the mode strength) value becomes larger at low temperatures, in contrast to our experiment. At the same time, our calculations are consistent with the available optical conductivity calculations in the $q$ quantum ${ }^{38-40}$ and classical ${ }^{32,34,35,41}$ approximations for an ion in the quartic potential. We explain the experimentally observed decrease in the strength of the $\mathrm{THz}$ soft mode at low temperature by its interaction with acoustic phonons (see, e.g., refs. ${ }^{42,43}$ ).

Making the final verdict regarding the microscopic mechanism of $\mathrm{THz}$ soft excitation needs more experimental and theoretical efforts. Note also that there are additional factors that can add to the observed exotic temperature variations of the soft mode frequencies and dielectric strengths, for instance, the rather complex structure of the studied compounds. Such complexity can make the soft mode couple to the magnetic subsystem (the possibility of spin-phonon coupling in hexaferrites has been reported in, e.g., refs. ${ }^{9,44-48}$ ). Additionally, we cannot exclude a coupling between the soft mode and the transitions within fine-structure components of the ${ }^{5} \mathrm{E}$ ground state of tetrahedrally coordinated $\mathrm{Fe}^{2+}$, since the frequencies of both types of excitations are close or even overlap, as discussed above (see Fig. 1).

One more aspect should be considered for the physics of the studied hexaferrites: there are indications that quantum critical phenomena can play a significant role in their dynamic properties. First principles calculations along with Monte Carlo simulations using the dipole-dipole interaction model ${ }^{7}$ indicate the possibility of a phase transition in $\mathrm{BaM}$ at $T_{\mathrm{c}}=3 \mathrm{~K}$ into a geometrically frustrated antiferroelectric state. Kumar and Pan$\mathrm{dey}^{49}$ claimed an observation of a phase transition in Casubstituted $\mathrm{BaM}$ at similar temperatures. They showed 
that the temperature of the phase transition rises with the concentration of the dopant in a manner characteristic of the phase transitions in a quantum critical regime. $\mathrm{Ca}^{2+}$ generates a positive chemical pressure within the $\mathrm{BaM}$ matrix, and as a result, it leads to a decrease in the unit cell parameters. The rise of the transition temperature $T_{\mathrm{c}}$ is connected with an increasing dipole-dipole interaction strength in and out of the $a b$-plane with shortening of bonds in the trigonal bipyramids due to the effect of positive chemical pressure. Accordingly, the positive chemical pressure can stabilize a quantum electric dipolar glass state and drive the system away from the quantum critical point ${ }^{49}$. It was further predicted that a negative chemical pressure should decrease the critical temperature of the transition and bring the substituted BaM closer to the quantum critical point. Negative chemical pressure can be produced in the $\mathrm{BaM}$ matrix by the substitution of $\mathrm{Ba}^{2+}$ with $\mathrm{Pb}^{2+}$, resulting in the $\mathrm{Ba}_{1-x} \mathrm{~Pb}_{x} \mathrm{Fe}_{12} \mathrm{O}_{19}$ materials studied here. According to our X-ray analysis (see Section SI1 in Supplemental Information), the addition of $\mathrm{Pb}^{2+}$ favors an expansion of the $a b$ face in the unit cell of the $\mathrm{Ba}_{1-x} \mathrm{~Pb}_{x} \mathrm{Fe}_{12} \mathrm{O}_{19}$ compounds, indicating that $\mathrm{Pb}^{2+}$ indeed generates a negative chemical pressure. When modeling the temperature dependences of the $\mathrm{THz}$ soft mode frequency by Eq. 2, the critical temperature $T_{\mathrm{c}}$ is lower in the compound with a larger content of $\mathrm{Pb}^{2+}$, in agreement with the suggestions of Kumar and Pandey ${ }^{49}$. Rowley et al. ${ }^{50}$ found evidence of the proximity of pure hexaferrites $\mathrm{BaM}$ and $\mathrm{SrFe}_{12} \mathrm{O}_{19}$ to the quantum critical point, which was indicated by a pronounced rise in the dielectric permittivity upon cooling. They also showed that, although pure $\mathrm{BaM}$ is expected to change its phase from paraelectric to antiferroelectric at approximately $6 \mathrm{~K}$, this transition is suppressed due to quantum fluctuations and does not occur at finite temperature. Evidence of the existence of quantum paraelectric phases in M-type hexaferrites was also reported in refs. ${ }^{7,12,13,49}$.

With the above taken into account, we consider the $\mathrm{THz}$ soft mode observed in the studied compounds as a possible fingerprint of a (quantum) phase transition that could happen in $\mathrm{Ba}_{1-x} \mathrm{~Pb}_{x} \mathrm{Fe}_{12} \mathrm{O}_{19}$, if not suppressed by quantum fluctuations, below the lowest temperatures of $4 \mathrm{~K}$ used in our experiments. It might be that doping with $\mathrm{Pb}$ destabilizes the coupled spin-phonon system in the material and brings it close to a quantum phase transition that can be driven by $\mathrm{Pb}$ doping, wherein the critical concentration can be close to $x(\mathrm{~Pb})=0.2$. This is evidenced by the critical temperatures obtained by fitting our data using Eq. 2, yielding a decrease from $T_{\mathrm{c}} \approx 3.3 \mathrm{~K}$ for $x=0.1$ to $T_{\mathrm{c}} \approx 1.5 \mathrm{~K}$ for $x=0.2$. It is known that, in the critical temperature region around the phase transition, when quantum effects come into play, the inverse dielectric constant of the corresponding soft excitation obeys a power law $(\Delta \varepsilon)^{-1} \sim T^{\gamma}$ with a critical exponent $\gamma=2$ for multiaxial dipole orientations when polarization is allowed to change along any direction ${ }^{51-53}$. Because we could not determine the soft mode dielectric strength precisely enough, we cannot make a firm conclusion on this theoretical prediction in the case of our hexaferrites. To clarify this matter, systematic and more detailed investigations on the spectroscopic properties of $\mathrm{Ba}_{1-x} \mathrm{~Pb}_{x} \mathrm{Fe}_{12} \mathrm{O}_{19}$ crystals with a series of $x(\mathrm{~Pb})$ and in both polarizations, $E \perp c$ and $E|| c$, are in progress.

\section{Phonon resonances in $\mathrm{Ba}_{1-x} \mathrm{~Pb}_{x} \mathrm{Fe}_{12} \mathrm{O}_{19}$}

For the $\mathrm{Ba}_{1-x} \mathrm{~Pb}_{x} \mathrm{Fe}_{12} \mathrm{O}_{19}, x=0.2$ sample, the spectrum of complex dielectric permittivity in the range from $1 \mathrm{~Hz}$ to $30 \mathrm{THz}\left(10^{-11}-10^{3} \mathrm{~cm}^{-1}\right)$ is shown in Fig. 1a, b. One can see that IR phonon resonances (sharp peaks $>80 \mathrm{~cm}^{-1}$ ) are nearly temperature independent down to $T=10 \mathrm{~K}$ : all IR spectra practically coincide at frequencies above $\approx 100 \mathrm{~cm}^{-1}$. The low-temperature drop in the intensity of the phonon peak at $\approx 90 \mathrm{~cm}^{-1}$ is caused by the softening of the $\mathrm{THz}$ absorption mode, as discussed above in Section 2.1. For an extended description of the IR phonon resonances for both compounds under study, see Supplemental Information, section SI2. The spectral response of the lattice vibrations observed in our compound is in line with the results obtained in other isostructural phases, such as pure $\mathrm{BaM}^{21}$ and $\mathrm{Pb}$-substituted $\mathrm{Ba}_{0.3} \mathrm{~Pb}_{0.7} \mathrm{Fe}_{12} \mathrm{O}_{19}{ }^{23}$.

\section{Radiofrequency and microwave relaxations}

Figure 1 clearly shows that the dielectric response of $\mathrm{Ba}_{1-x} \mathrm{~Pb}_{x} \mathrm{Fe}_{12} \mathrm{O}_{19}, x=0.2$, compound at low frequencies, $1 \mathrm{~Hz}-300 \mathrm{MHz}$, strongly depends on both frequency and temperature. A similar behavior was found for the $x=0.1$ compound. The multiple steps in $\varepsilon^{\prime}(v)$ and the peaks and corresponding shoulders in the $\varepsilon^{\prime \prime}(v)$ spectra indicate a rather complex relaxational behavior. The spectra at the highest temperature shown $(297 \mathrm{~K})$ reveal a noticeable mismatch between the results from the frequency response $(v \lesssim 1 \mathrm{MHz}$; open circles) and those from the reflectometric techniques $(v \gtrsim 1 \mathrm{MHz}$; closed circles), performed with the different types of electrodes and sample geometries. This points to an extrinsic origin of the relaxations observed at high temperatures ${ }^{54}$, as discussed in detail below. However, the limiting plateau value on the order of $\varepsilon^{\prime}=30$, approached at low temperature and high frequencies, should be of intrinsic nature, and it reflects the ionic and electronic polarizability of the material (termed $\varepsilon_{\infty}$ in dielectric spectroscopy). Its offset (on the order of 20) compared to the low-frequency values of the $\mathrm{THz}$ data indicates additional processes occurring in the frequency gap between $10^{-2}$ and $4 \mathrm{~cm}^{-1}$.

The low-frequency dielectric spectra are shown in more detail in Fig. 3, where it is seen that there are at least three 


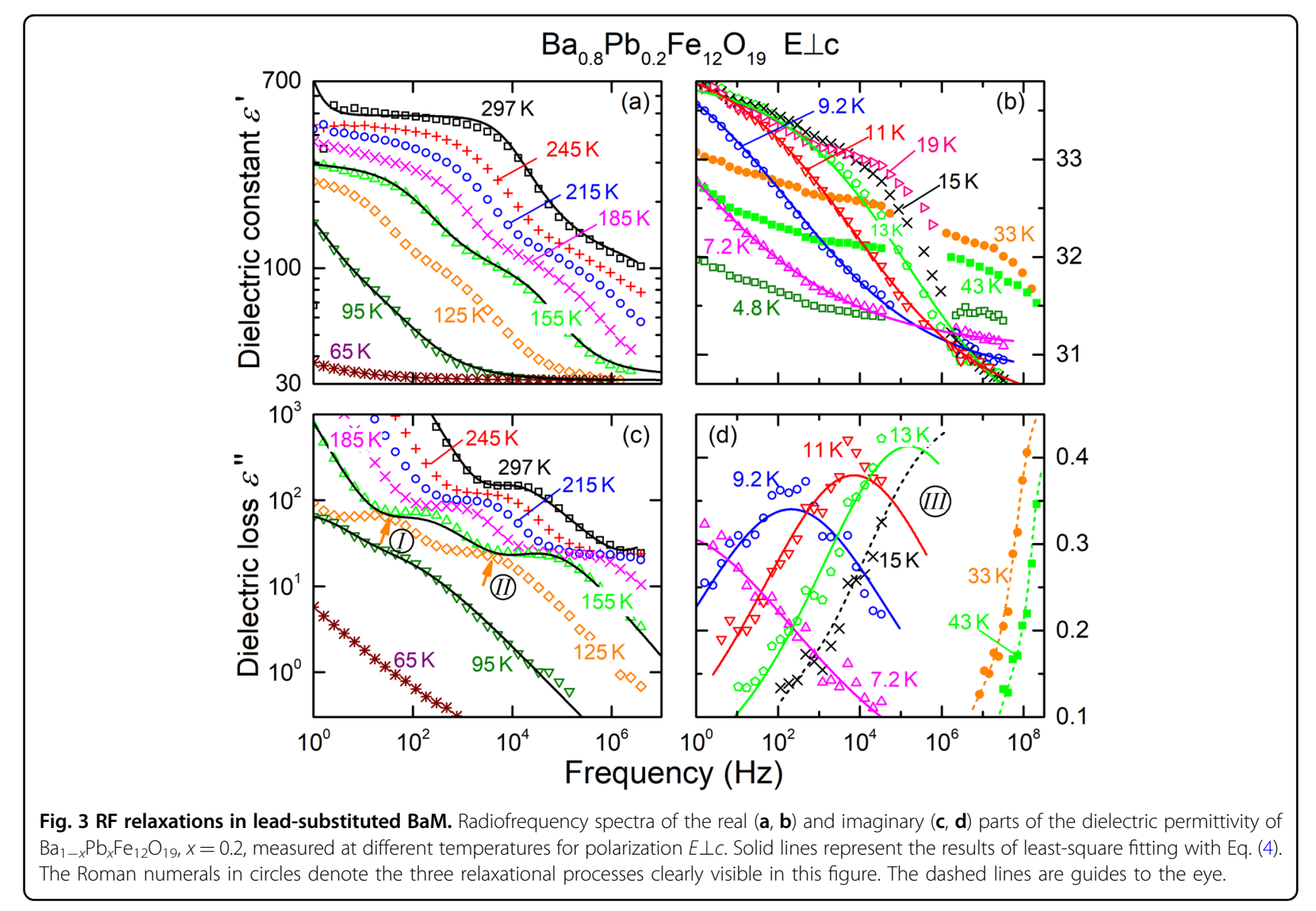

temperature-dependent relaxational processes in the radiowave and microwave ranges. Upon cooling from room temperature down to $60-70 \mathrm{~K}$, two relaxational loss peaks (marked as I and II in Fig. 3c) pass through the frequency window of our experiment $(1 \mathrm{~Hz}-4 \mathrm{MHz})$, accompanied by the corresponding step-like features in the spectra of the real permittivity $\varepsilon^{\prime}(v)$ (Fig. 3a). A third process (termed III) enters the experimental window when the temperature is lowered down to approximately $40 \mathrm{~K}$, and it removes the window at $T<7 \mathrm{~K}$ (Fig. 3b, d). In contrast to processes I and II (cf. Fig. 1), its behavior as detected by frequency-response analysis (up to approximately $1 \mathrm{MHz}$ ) is consistent with that detected by the reflectometric technique (beyond $1 \mathrm{MHz}$, see Fig. 3b), pointing to an intrinsic origin. In the spectra at $T=15$ and $19 \mathrm{~K}$ (Fig. 3b), a small additional $\varepsilon^{\prime}(v)$ step is superimposed on the main relaxation at low frequencies. This indicates a fourth process, discussed in more detail below. At 33 and $43 \mathrm{~K}$, only the low-frequency wings of the loss peaks are detected (Fig. 3d). Employing a closed-cycle refrigerator with shorter coaxial lines allowed reliable measurements up to approximately $200 \mathrm{MHz}$; however, the temperature was limited to $33 \mathrm{~K}$.
The three relaxation features were modeled using the empirical Havriliak-Negami expression for the complex dielectric permittivity (solid lines in Fig. 3):

$$
\varepsilon^{*}(\nu)=\varepsilon^{\prime}(\nu)+i \varepsilon^{\prime \prime}(\nu)=\sum_{k} \frac{\Delta \varepsilon_{k}}{\left(1+\left(i 2 \pi \nu \tau_{k}\right)^{1-\alpha}\right)^{\beta}} .
$$

Here $\Delta \varepsilon_{k}$ is the dielectric strength of the $k$-th relaxation; $\tau_{k}$ is the mean relaxation time of the relaxation process, and $\alpha$ and $\beta$ are coefficients describing the broadening and asymmetry of the relaxational spectral response, respectively; their temperature dependences are presented in Fig. SI6-1 in the Supplementary Information. The fits were only performed for temperatures wherein peak frequencies (in $\varepsilon^{\prime \prime}$ ) and/or points of inflection (in $\varepsilon^{\prime}$ ) could be clearly discerned in the spectra.

The relaxation processes are often revealed more clearly in temperature-dependent plots of the permittivity, especially if the relaxations appear close to the device operation limit, as is the case for process III (cf. Fig. 3d). Figure 4a, b displays the temperature dependences of the real $\varepsilon^{\prime}$ and imaginary $\varepsilon^{\prime \prime}$ parts of the dielectric permittivity measured at 


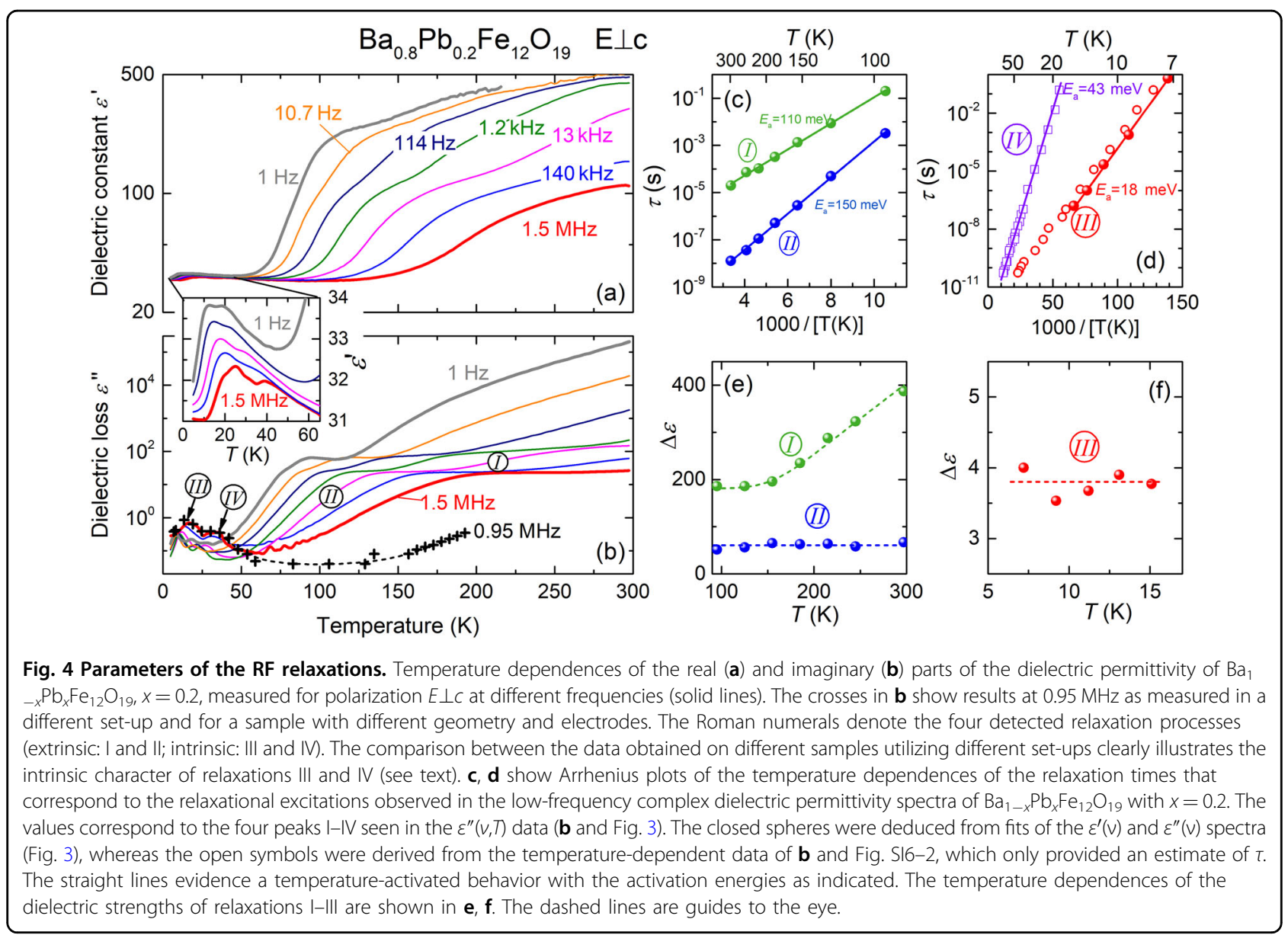

various fixed frequencies (solid lines). These plots reveal four pronounced anomalies with shapes typical of relaxational processes (peaks in $\varepsilon^{\prime \prime}$ and steps in $\varepsilon^{\prime}$ ). Their frequency-dependent character is better visible in a plot of the temperature-dependent AC conductivity $\sigma^{\prime}(T)$ (Fig. SI6-2), wherein the different curves exhibit less overlap due to the offset caused by the relation $\sigma^{\prime}(T) \sim v \varepsilon^{\prime \prime}(T)$. The inset of Fig. 4b provides a magnified view of $\varepsilon^{\prime}(T)$ for the two lowtemperature relaxations, revealing a weak but significant increase in their static real permittivity with decreasing temperature, in accordance with the temperaturedependent static dielectric constant becoming obvious in Fig. 3b. Comparing the frequencies and temperatures of the occurrence of these four processes with those in Fig. 3, three of them can be clearly identified with relaxations I, II, and III, discussed above. The features emerge when, during cooling, the relaxational peaks in $\varepsilon^{\prime \prime}(v)$ and the corresponding points of inflection in the $\varepsilon^{\prime}(v)$ spectra of processes I, II, and III shown in Fig. 3 move into the measurement frequency interval. Interestingly, in Fig. 4, a weak additional process (IV) is detected between approximately 20 and $40 \mathrm{~K}$ and depends on the frequency. We note that the temperature-dependent plots in Fig. 4 point to the absence of any phase transition at low temperatures down to $T \approx 2 \mathrm{~K}$, which is also evident from the smooth temperature dependence of the specific heat (see Fig. SI7-1 in the Supplementary Information) and from IR spectra (Figs. $1 \mathrm{c}$ and SI2-2) where no extra (folding) phonons were observed that would indicate structural changes in the lattice.

The pluses in Fig. 4b represent $\varepsilon^{\prime \prime}(T)$ results for $0.95 \mathrm{MHz}$ as measured independently in a special experimental set-up (Using a Janis He-flow cryostat in Prague instead of the Cryovac He-bath cryostat in Augsburg) for another sample with different geometries and contacts. The agreement in the region of relaxations III and IV clearly points to their intrinsic origin. In contrast, marked differences in the region of high-temperature relaxations I and II are observed, which we also found for various other measurements performed with different set-ups and for various samples with particular types of electrodes. As already suggested above based on Fig. 1, this proves the extrinsic, most likely contact-related origin of these processes. It is well known that extrinsic, so-called Maxwell-Wagner relaxations can arise, e.g., due to the formation of Schottky barriers at the sample-electrode 
interfaces, which leads to deviations, as observed in the present case ${ }^{54}$.

The temperature-dependent results of Fig. 4 (and in Fig. SI6-2) provide an additional way to estimate relaxation times as a function of temperature by determining the loss peak temperatures $T_{\mathrm{p}}$ for a given measurement frequency $v$. For $T=T_{\mathrm{p}}$, the relaxation time is given by $\tau(T)=1 /$ $(2 \pi v)$. This enables an extension of the covered temperature and frequency range for $\tau(T)$, albeit with a somewhat reduced precision compared with the relaxation times deduced from fits of the frequency-dependent data (Figs. 1 and 3). Figure 4c, d give an Arrhenius presentation of the relaxation times $\tau$ of the four detected processes. The $\tau(T)$ data follow an activated behavior with four activation energies: $110 \mathrm{meV}$ (process I), $150 \mathrm{meV}$ (process II), $18 \mathrm{meV}$ (process III), and $43 \mathrm{meV}$ (process IV). According to Fig. 4e, f, the dielectric strengths $\Delta \varepsilon$ of relaxations II and III do not change with temperature in the shown temperature intervals. The dielectric strength of relaxation I reveals a strong decrease upon cooling down to $150 \mathrm{~K}$, but it levels off at lower temperatures. The dielectric strength of process IV is too weak to allow for any unequivocal determination of its temperature dependence. The $\Delta \varepsilon$ results for process III (Fig. 4f) are also of limited precision because of the superimposed contribution of process IV, which was not considered in the fits.

Whereas relaxations I and II are extrinsic, intrinsic relaxations III and IV might manifest the dynamic response of dielectric or magnetic domains, similar to a related compound with a higher concentration of $\mathrm{Pb}$, $\mathrm{Ba}_{0.3} \mathrm{~Pb}_{0.7} \mathrm{Fe}_{12} \mathrm{O}_{19}{ }^{23}$. In that compound, pronounced dielectric and magnetic relaxational dynamics were detected in the radiofrequency range; both relaxations exhibited similar characteristic relaxation times, evidencing the birelaxor-like nature of $\mathrm{Ba}_{0.3} \mathrm{~Pb}_{0.7} \mathrm{Fe}_{12} \mathrm{O}_{19}{ }^{23}$. Our magnetic/electrostatic force microscopic experiments clearly indicate the presence of magnetic domains in pure BaM (Fig. SI8-1, SI8-2, SI8-3 in Supplementary Information). Thus, relaxation-like dispersion was observed in the magnetic susceptibility spectra of $\mathrm{Ba}_{0.8} \mathrm{~Pb}_{0.2} \mathrm{Fe}_{12} \mathrm{O}_{19}$ (Fig. SI9-1, SI9-2) should be associated with magnetic domain dynamics. Regarding dielectric relaxations III and IV, we suggest that their origin should be related to the response of magnetic domain walls, which are electrically polar in the present compound. The mechanism of the emergence of electric polarization in magnetic domain walls is analyzed in ref. ${ }^{55}$ taking iron garnet as an example. We firmly detect the electrical polarity at the boundaries of magnetic domains in undoped BaM (Fig. $\mathrm{SI} 8-2)$. At the same time, in $\mathrm{Pb}$-doped $\mathrm{Ba}_{0.8} \mathrm{~Pb}_{0.2} \mathrm{Fe}_{12} \mathrm{O}_{19}$, along with the clearly visible magnetic domain structure (Fig. SI8-3), we were not able to detect any sign of the domain polarity, which is most likely beyond the sensitivity of the technique used. This last suggestion is confirmed by the low values of dielectric strengths of the two relaxations, III and IV, $\Delta \varepsilon<\approx 10$, which is by orders of magnitude less than those seen in pure $\operatorname{BaM}(\Delta \varepsilon \approx$ $1000)^{23}$. We also note a considerable difference in the activation energies of these relaxations, $18 \mathrm{meV}$ (III) and $43 \mathrm{meV}$ (IV).

\section{Gigahertz resonances}

Although our experiments did not cover the spectral range between several $\mathrm{GHz}$ and $\sim 100 \mathrm{GHz}$, our measurements indicate that some excitations exist in this frequency interval. First, this is evidenced by a "step" in the spectra of the real permittivity between the radiofrequencies and $\mathrm{THz}$ frequencies, as seen in Fig. 1a. In addition, below $20 \mathrm{~cm}^{-1}$, there is a clear decrease in the real part of permittivity $\varepsilon^{\prime}(v)$ and, below $10 \mathrm{~cm}^{-1}$, an increase in its imaginary part $\varepsilon^{\prime \prime}(v)$ (see Fig. SI4-1). Both of these features are linked together, and they strongly suggest the existence of a resonant absorption band located below $3 \mathrm{~cm}^{-1}(90 \mathrm{GHz})$. Assuming its purely dielectric nature, we were able to apply expression (1) to model its spectral response with a dielectric Lorentzian, which let us estimate the values and the temperature dependences of its parameters. Examples of the corresponding least-square fits of the room-temperature spectra are given in Fig. SI4-1. As demonstrated in Fig. SI4-2, there are no strong changes with temperature in the frequency position and damping parameter of the excitation; additionally, its dielectric and oscillator strengths show signs of a decrease in cooling. Note that the dielectric strength of the resonance practically matches the "step" between the radiofrequency and $\mathrm{THz}$ values of real permittivity (Fig. 1a), signifying that there are no additional absorption bands in the range except the one at $2-3 \mathrm{~cm}^{-1}$. For the microscopic origin of the detected $\mathrm{GHz}$ band, we cannot exclude that it can be of mixed magnetoelectric origin ${ }^{56,57}$. Some evidence for that is given by the coincidence (within experimental uncertainties) of its frequency position with the value of the frequency of FMR in the $\mathrm{Ba}_{0.9} \mathrm{~Pb}_{0.1} \mathrm{Fe}_{12} \mathrm{O}_{19}$ crystal obtained by the extrapolation of the magnetic fielddependent frequency of the resonance to zero magnetic field (Fig. SI10-1); the oscillator strength of the FMR remains constant (Fig. SI10-2).

\section{Conclusions and prospects for use in $\mathrm{THz}$ electronics}

Our thorough spectroscopic studies of the broad-band ( $1 \mathrm{~Hz}$ up to $240 \mathrm{THz})$ temperature-dependent $(5-300 \mathrm{~K})$ electromagnetic properties of $\mathrm{Ba}_{1-x} \mathrm{~Pb}_{x} \mathrm{Fe}_{12} \mathrm{O}_{19}$ single crystals found a rich variety of strongly temperature- and doping-dependent electric, magnetic, and possibly magnetoelectric excitations. Most intriguing from both 
fundamental and applied points of view is the complex electromagnetic response of the compounds at $\mathrm{THz}$ frequencies that is determined by electronic transitions between the fine-structure components of the ${ }^{5} \mathrm{E}$ ground state of tetrahedrally coordinated $\mathrm{Fe}^{2+}$ ions, which overlap with a soft excitation whose temperature evolution resembles that of a ferroelectric soft mode. A close inspection reveals, however, that the temperature dependences of the resonance frequency and strength do not follow the corresponding Cochran and Curie-Weiss laws known to describe soft modes in classical ferroelectrics. We proposed microscopic models that qualitatively explain the observed nonstandard behavior of the excitation. The radiofrequency electrodynamic characteristics of the studied compounds are fully determined by relaxational processes that are related to the Maxwell-Wagner polarization and to the dynamics of magnetic domain walls. Our findings are an important step toward a deep understanding of the physics behind the electromagnetic response of the studied hexaferrites from radiofrequency all the way up to $\mathrm{THz}$ and IR.

A few words on applicational aspects of the studied materials are in order. The rapidly developing field of forthcoming $\mathrm{THz}$ electronics motivates the search for materials that satisfy the ever-increasing requirements; see, e.g., ref. ${ }^{58}$ and references therein ${ }^{59}$. With the results presented here, we demonstrate that hexaferrites are among the most promising candidates. Being planar, selfbiased, and low-loss materials, hexaferrites meet the requirements for the development of microwave and $\mathrm{THz}$ circulators, phase shifters, filters, isolators, and millimeter-wave resonator antennas ${ }^{15,16,56,60}$. These compounds are considered one of the most promising material classes for telecommunication systems ${ }^{61}$. In ref. ${ }^{61}$, it was shown that a $\mathrm{BaFe}_{12} \mathrm{O}_{19}$-polydimethylsiloxane nanocomposite exhibits a zero-bias (no external magnetic field) FMR at a frequency of $46.6 \mathrm{GHz}$ and is thus proposed for the design of a circulator with an operating frequency of $35 \mathrm{GHz}$. The absorption resonances at even higher frequencies of approximately 50 and $100 \mathrm{GHz}$ that we discovered in the present study in single crystalline $\mathrm{Pb}$-doped hexaferrites $\mathrm{Ba}_{1-x} \mathrm{~Pb}_{x} \mathrm{Fe}_{12} \mathrm{O}_{19}$ make these compounds promising candidates for manufacturing self-biased devices with working frequencies meeting standards of higher generations in telecommunications. Considerations of the possibilities of the intentional tuning of the resonance frequency with dopant content $x$ or (moderate) external magnetic fields are in progress, as well as the characterization of ceramic, sputtered films and nanocomposite forms of the compounds. Metamaterials and composites based on hexaferrites are also used to develop circulators for 5G technology ${ }^{62,63}$, low-loss sub-THz isolators, resonators, filters, and phase shifters ${ }^{64}$. Hexaferrite-piezoelectric and hexaferrite-ferroelectric composites have been proposed for tunable devices to control $\mathrm{THz}$ radiation ${ }^{65}$, particularly for dual electric and magnetic field tunable resonators, filters, and phase shifters operating at $\mathrm{THz}$ frequencies. Dielectric anisotropy of BaM is employed in millimeter-wave resonators ${ }^{56,66}$. There are still some problems to overcome. For example, when thin films of lead-substituted barium hexaferrite are used, one has to sustain a proper and homogeneous substitution level over the film surface, as lead substitutes barium in the crystal lattice rather chaotically. Such a problem is not inherent in bulk materials owing to the averaging of the lead content throughout the volume.

Information on the broad-band electronic and/or dielectric properties of hexaferrites is also in demand when developing devices based on monitoring the direct current resistivity or AC impedance of thin films of diverse morphologies. One of the hottest and most promising areas today includes the development of chemical or biological sensors; see, e.g., refs. ${ }^{67-69}$. Additionally, in this area, barium hexaferrite and corresponding substituted compounds reveal encouraging results. Corresponding perspectives include ozone sensors ${ }^{70}$, ammonia sensors ${ }^{71}$, and acetone and ethanol sensors ${ }^{72}$. Considering ammonia detection, sensors based on hexaferrites ${ }^{71}$ reveal much higher sensitivity (lowest targeted gas concentration) than $\mathrm{MO}_{3}$-based sensors ${ }^{69}$.

The growth of high-quality single crystals with desirable (enhanced) size remains a challenge. Methods to synthesize powder, ceramic, and polycrystalline forms of hexaferrites are well developed. However, fundamental studies and specific applications (e.g., optically active media, polarizers, phase shifters) require relatively large single crystals with reproducible properties independent of the growth technique, a feature that is not inherent to noncrystalline forms of the material. On the other hand, there are a number of advantages that make $\mathrm{Ba}-\mathrm{Pb}$ hexaferrites attractive. They are nontoxic (even despite the presence of toxic lead in the chemical formula) and corrosion-stable. They are cheap to produce since the oxides needed in the synthesis are waste from machinery fabrication, which in turn makes production eco-friendly. Although the production of hexaferrites is a billion dollar income business, they are currently used mainly in the microwave magnetic device market. The lack of information on $\mathrm{THz}$ characteristics discourages their wide application for the development of forthcoming $\mathrm{THz}$ devices. At the same time, the number of yearly publications on the $\mathrm{THz}$ physics of hexaferrites is growing rapidly, which will trigger their use in the electronic industry. In this respect, a significant result of the present work is the quantitative information on the electrodynamic properties of hexaferrites in an extremely wide frequency range, including bands that are most attractive 
from the viewpoint of the development of next-generation subTHz-THz communication systems and the fabrication of $\mathrm{THz}$-tunable electronic components, such as band-stop filters, phase shifters, and attenuators, surpassing existing analogs in their characteristics ${ }^{56,73,74}$. We have unveiled the fundamental physical mechanisms that determine these properties. Taking into account the multiferroic properties of barium hexaferrites ${ }^{75-77}$, the area of their possible applications can expand remarkably.

\section{Methods}

\section{Sample preparation}

Single crystals of $\mathrm{Pb}$-substituted barium hexaferrite with chemical compositions $\mathrm{Ba}_{1-x} \mathrm{~Pb}_{x} \mathrm{Fe}_{12} \mathrm{O}_{19}(x=0.1$ and 0.2) were grown using the flux method. Crystal growth and characterization are described in section SI1 of the Supplemental Information. For spectroscopic $\mathrm{THz}$ and IR measurements, the crystals were carefully oriented (see section SI1 in Supplemental Information) and then prepared as plane-parallel (to within a few micrometers) plates with the crystallographic $c$-axis oriented perpendicular to plate surfaces; this geometry allowed for measurements with the linear polarization of the electric field E-vector of the probing radiation lying within the $a b$ planes, $E \perp c$. Both sides of the prepared plates were carefully polished with diamond lapping films to meet the requirements of THz-IR measurements. The sizes of the samples for $\mathrm{THz}$ and IR measurements were approximately $3 \mathrm{~mm} \times 4 \mathrm{~mm}$ with thicknesses of $335 \mu \mathrm{m}(x=0.1)$ and $452 \mu \mathrm{m}(x=0.2)$. For measurements at frequencies from $1 \mathrm{~Hz}$ up to the microwave range $(1 \mathrm{GHz})$, specimens were fabricated as thin plane-parallel polished plates with thicknesses of $174 \mu \mathrm{m}$ (Augsburg) and $758 \mu \mathrm{m}$ (Prague) with the $c$-axis parallel to the plate surfaces, thus allowing for measurements in the $E \perp c$ geometry.

\section{THz-IR spectroscopy}

Experiments in the $\mathrm{THz}$ range (at frequencies $v=8-100 \mathrm{~cm}^{-1}, 0.24-3 \mathrm{THz}$ ) were conducted with the help of two time-domain spectrometers, Menlo Tera K15 and TeraView TPS 3000. The spectra of the complex transmission coefficient (amplitude and phase) of the plane-parallel samples were measured, and the spectra of complex dielectric permittivity $\varepsilon^{*}(v)=\varepsilon^{\prime}(v)+i \varepsilon^{\prime \prime}(v)$ were calculated using standard Fresnel equations ${ }^{78}$. In the farIR and mid-IR spectral regions $\left(30-8000 \mathrm{~cm}^{-1}\right.$, 0.9-240 THz), a standard Fourier transform spectrometer Bruker Vertex 80 was utilized for the measurements of reflectivity spectra. To measure reflectivity at the lowest frequencies $\left(25-80 \mathrm{~cm}^{-1}\right)$, a Bruker 113v Fourier transform spectrometer with a helium-cooled bolometer was used. The experiments were performed over a wide range of temperatures (5-300 K) using exchange-gas and coldfinger cryostats. For the experiments in external magnetic fields of up to $7 \mathrm{~T}$, Spectromag Oxford cryomagnets attached to a Fourier transform IR spectrometer Bruker $113 \mathrm{v}$ and to a custom-made time-domain $\mathrm{THz}$ spectrometer were employed.

\section{Radio frequency impedance spectroscopy}

At frequencies from $\approx 1 \mathrm{~Hz}$ to $\approx 200 \mathrm{MHz}$ and at temperatures from $5 \mathrm{~K}$ to $300 \mathrm{~K}$, the dielectric response of the samples was measured using a Novocontrol Alpha frequency-response analyzer and a Keysight 4991B impedance analyzer. For cooling, a closed-cycle refrigerator (CTI-Cryogenics), a He-flow cryostat (JANIS ST100), and a He-bath cryostat (Cryovac) were used. For the measurements at frequencies of up to approximately $4 \mathrm{MHz}$, Pt-Au electrodes were evaporated using a Bal-Tex SCD 050 sputter coater onto the principal faces of the samples or silver paste layers were directly applied as electrodes. The connections for applying the AC electric field were provided by silver or copper wires fixed to the electrodes by silver paste. For frequencies above approximately $1 \mathrm{MHz}$, a coaxial reflectometric set-up was used, employing the impedance analyzer as described in ref. ${ }^{79}$. Here the sample was mounted between two metal plates at the end of a coaxial line. Depending on the cooling devices used, the dielectric response of the samples was measured in vacuum or helium atmospheres, which did not significantly influence the measured dielectric properties. The precise determination of the absolute values of the complex dielectric permittivity is hampered by the occurrence of stray capacitances and geometrical uncertainties, which play a role, especially for rather small single crystals, as investigated in the present work. For the frequency-response measurements below approximately $4 \mathrm{MHz}$, the largest samples were available. Here different samples with different geometries and contact configurations were independently measured by the Prague and Augsburg groups, yielding comparable absolute values in the frequency/temperature ranges dominated by intrinsic effects. The less precise highfrequency results obtained by the reflectometric method were scaled to the low-frequency results by a single factor for all data obtained during a measurement run.

\section{Heat capacity measurements}

The temperature dependence of the heat capacity $c_{\mathrm{P}}$ was measured (via the standard relaxation method) using the heat capacity option of the Physical Property Measurement System (Quantum Design).

\section{Magnetic force microscopy}

With scanning probe microscopy, we investigated two samples, $\mathrm{BaFe}_{12} \mathrm{O}_{19}$ and $\mathrm{Ba}_{1-x} \mathrm{~Pb}_{x} \mathrm{Fe}_{12} \mathrm{O}_{19}(x=0.2)$, to prove the multiferroic nature of the crystals. We used magnetic force microscopy to observe the magnetic 
domain structure under low temperature $(4.5 \mathrm{~K})$ and ambient conditions. For low-temperature measurements, the AttoDRY 1000 system with the possibility of applying a magnetic field was used. For room-temperature investigations, we used the NT-MDT AFM device. These magnetic force measurements were performed with a Bruker MESP-V2 cantilever. Both crystals show similar domain structures with negligible temperature dependences and a clear magnetic response to the external magnetic field. After that, we used a conductive (PtIr coated, Bruker) cantilever to check the electrostatic response of the samples. In BaM, an electrostatic polarization of magnetic domain boundaries is clearly observed, whereas in the Pb-doped crystal, no such effect was detected.

\section{Acknowledgements}

The authors are grateful to Professor A. Mukhin and Professor G. Komandin for fruitful discussions. The THz cryogenic study was supported by the Russian Science Foundation, grant 19-72-00055; IR measurements were supported by the RFBR (grant 20-32-90034), LNA acknowledges support of the Russian Ministry of Higher Education and Science, state contract 1740-20 and system of President's scholarships for young researchers (SP-777.2021.5). Czech coauthors were supported by the Czech Science Foundation (Project No. 21-06802S) and by the MŠMT Projects LM2018096 and SOLID21-CZ.02.1.01/ $0.0 / 0.0 / 16 \_019 / 0000760$. Single crystal growth was supported at SUSU by the RFBR (grant 20-08-00716), and DAV was partially supported by President's grants for young doctors of science (MD-5612.2021.4). A theoretical study at IA\&E SB RAS was supported by RFBR (grants 20-02-00314 and 20-42-540002). Work done at the University of Augsburg was supported by the DFG via the TRR 80 (ID 107745057).

\section{Author details}

'Laboratory of Terahertz Spectroscopy, Center for Photonics and 2D Materials, Moscow Institute of Physics and Technology, Dolgoprudny, Russia. ${ }^{2}$ Prokhorov General Physics Institute of the Russian Academy of Sciences, 119991 Moscow, Russia. ${ }^{3}$ South Ural State University, 454080 Chelyabinsk, Russia. ${ }^{4}$ Department of Physics, Faculty of Science, Sohag University, Sohag University Street, Sohag, Egypt. ${ }^{5}$ Southern Federal University, 344006 Rostov-on-Don, Russia. Institute of Physics of the Czech Academy of Sciences, 18221 Praha 8, Czech Republic. ${ }^{7}$ Faculty of Mathematics and Physics, Charles University, 12116 Prague 2, Czech Republic. ${ }^{8}$ 1. Physikalisches Institut, Universität Stuttgart, 70550 Stuttgart, Germany. ${ }^{9}$ Institute of Automation and Electrometry SB RAS, 630090 Novosibirsk, Russia. ${ }^{10}$ Experimental Physics V, Center for Electronic Correlations and Magnetism, University of Augsburg, 86159 Augsburg, Germany

\section{Author contributions \\ L.N.A. conceived, planned, and performed the experiments, data analysis and interpretation of the results and wrote the paper in consultation with A.S.P., P. L., and B.P.G.; D.A.V. provided single crystals and their characterization; V.B.A., C. K., F.K., J.P., S.K., M.D., and A.V.P. provided access to the facility; A.G.A., A.M., P.B., F.K., C.K., E.d.P., P.P., A.V.P., and S.S. assisted in the experiments; V.A.A. provided theoretical model; V.V.D. performed EFM/MFM experiments; M.S. and P.L. held RF experiments, and P.L. wrote the RF part of the manuscript; B.P.G. analyzed the results, managed and advised the research, and edited the manuscript. All authors reviewed the manuscript.}

\section{Competing interests}

The authors declare no competing interests.

\section{Publisher's note}

Springer Nature remains neutral with regard to jurisdictional claims in published maps and institutional affiliations.
Supplementary information The online version contains supplementary material available at https://doi.org/10.1038/s41427-021-00331-x.

Received: 7 May 2021 Revised: 10 August 2021 Accepted: 23 August 2021 Published online: 17 September 2021

\section{References}

1. Sakai, K. \& Tani, M. in Terahertz Optoelectronics 1-30 (Springer-Verlag, 2005).

2. Aghasi, H. et al. Terahertz electronics: application of wave propagation and nonlinear processes. Appl. Phys. Rev. 7, 021302 (2020).

3. Romanczyk, B. et al. W-band power performance of SiN-passivated N-polar GaN deep recess HEMTs. IEEE Electron Device Lett. 41, 349-352 (2020).

4. Song, H.-J. \& Nagatsuma, T. Present and future of terahertz communications. IEEE Trans. Terahertz Sci. Technol. 1, 256-263 (2011).

5. Tonouchi, M. Cutting-edge terahertz technology. Nat. Photonics 1, 97-105 (2007).

6. Williams, B. S. Terahertz quantum-cascade lasers. Nat. Photonics 1, 517-525 (2007).

7. Wang, P. S. \& Xiang, H. J. Room-temperature ferrimagnet with frustrated antiferroelectricity: promising candidate toward multiple-state memory. Phys. Rev. X 4, 011035 (2014).

8. Kimura, T. Magnetoelectric hexaferrites. Annu. Rev. Condens. Matter Phys. 3, 93-110 (2012).

9. Laguta, V. et al. Magnetoelectric coupling in multiferroic Z-type hexaferrite revealed by electric-field-modulated magnetic resonance studies. J. Mater. Sci. 55, 7624-7633 (2020).

10. Zhai, K et al. Giant magnetoelectric effects achieved by tuning spin cone symmetry in Y-type hexaferrites. Nat. Commun. https://doi.org/10.1038/ s41467-017-00637-x (2017).

11. Chun, S. H. et al. Electric field control of nonvolatile four-state magnetization at room temperature. Phys. Rev. Lett. 108, 177201 (2012).

12. Shen, S.P. et al. Quantum electric-dipole liquid on a triangular lattice. Nat. Commun. 7, 10569 (2016).

13. Shen, S.P. et al. Magnetic-ion-induced displacive electric polarization in $\mathrm{FeO}_{5}$ bipyramidal units of ( $\mathrm{Ba}, \mathrm{Sr}) \mathrm{Fe}_{12} \mathrm{O}_{19}$ hexaferrites. Phys. Rev. B 90, 180404 (2014).

14. Harris, V. G. Modern microwave ferrites. IEEE Trans. Magn. 48, 1075-1104 (2012).

15. Zuo, X., How, H., Somu, S. \& Vittoria, C. Self-biased circulator/isolator at millimeter wavelengths using magnetically oriented polycrystalline strontium M-type hexaferrite. In IEEE Transactions on Magnetics 3160-3162 (IEEE, 2003).

16. Song, Y.-Y. Y., Sun, Y., Lu, L., Bevivino, J. \& Wu, M. Self-biased planar millimeter wave notch filters based on magnetostatic wave excitation in barium hexagonal ferrite thin films. Appl. Phys. Lett. 97, 173502 (2010).

17. Harris, V. G. et al. Recent advances in processing and applications of microwave ferrites. J. Magn. Magn. Mater. https://doi.org/10.1016/j. jmmm.2009.01.004 (2009).

18. Pullar, R. C. Hexagonal ferrites: a review of the synthesis, properties and applications of hexaferrite ceramics. Prog. Mater. Sci. 57, 1191-1334 (2012).

19. Gorbachev, E. A. et al. Hexaferrite materials displaying ultra-high coercivity and sub-terahertz ferromagnetic resonance frequencies. Mater. Today 32, 13-18 (2020).

20. Gudkov, V. V. et al. Sub-lattice of Jahn-Teller centers in hexaferrite crystal. Sci. Rep. 10, 7076 (2020).

21. Mikheykin, A. S. et al. Lattice anharmonicity and polar soft mode in ferrimagnetic M-type hexaferrite $\mathrm{BaFe}_{12} \mathrm{O}_{19}$ single crystal. Eur. Phys. J. B 87, 232 (2014).

22. Zhukova, E. S. et al. Crucial influence of crystal site disorder on dynamical spectral response in artificial magnetoplumbites. Solid State Sci. 62, 13-21 (2016).

23. Alyabyeva, L. N. et al. Influence of chemical substitution on broadband dielectric response of barium-lead M-type hexaferrite. N. J. Phys. 21, 063016 (2019).

24. Ahmed, A. et al. Effect of aluminium substitution on low energy electrodynamics of barium-lead M-type hexagonal ferrites. J. Phys. Conf. Ser. 1389, 012044 (2019).

25. Alyabyeva, L. et al. Terahertz-infrared electrodynamics of lead-doped single crystalline $\mathrm{Ba}_{1-x} \mathrm{~Pb}_{x} \mathrm{Fe}_{12} \mathrm{O}_{19} \mathrm{M}$-type hexagonal ferrite. in Proc. 43rd International Conference on Infrared, Millimeter, and Terahertz Waves, IRMMW-THz 18227333 (IEEE, 2018). 
26. Alyabyeva, L. et al. Terahertz-infrared spectroscopy of Ti4+-doped M-type barium hexaferrite. J. Alloy. Compd. 820, 153398 (2020).

27. Kadlec, F. et al. Electromagnon in the Z-type hexaferrite $\left(\mathrm{Ba}_{x} \mathrm{Sr}_{1-x}\right)_{3} \mathrm{Co}_{2} \mathrm{Fe}_{24} \mathrm{O}_{41}$. Phys. Rev. B 94, 024419 (2016)

28. Vit, J. et al. Electromagnon in the Y-type hexaferrite $\mathrm{BaSrCoZnFe}{ }_{11} \mathrm{AlO}_{22}$. Phys. Rev. B 97, 134406 (2018)

29. Lines, M. E., Glass, A. M. \& Burns, G. Principles and applications of ferroelectrics and related materials. Phys. Today 31, 56-58 (1978).

30. Kamba, S. Soft-mode spectroscopy of ferroelectrics and multiferroics: a review. APL Mater. 9, 020704 (2021).

31. Obradors, X., Collomb, A., Pernet, M., Samaras, D. \& Joubert, J. C. X-ray analysis of the structural and dynamic properties of $\mathrm{BaFe}_{12} \mathrm{O}_{19}$ hexagonal ferrite at room temperature. J. Solid State Chem. 56, 171-181 (1985).

32. Nakajima, Y. \& Naya, S. Orientational phase transition and dynamic susceptibility of hindered-rotating dipolar system -a librator-rotator model-. J. Phys. Soc. Jpn. 63, 904-914 (1994).

33. Normand, B. G. A.r Giddy, A. P., Dove, M. T. \& Heine, V. Bifurcation behaviour in structural phase transitions with multi-well potentials. J. Phys. Condens. Matter 2, 3737-3745 (1990).

34. Onodera, Y. Dynamical response of ferroelectrics in terms of a classical anharmonic-oscillator model. J. Phys. Soc. Jpn. 73, 1216-1221 (2004).

35. Gorshunov, B. P. et al. Incipient ferroelectricity of water molecules confined to nano-channels of beryl. Nat. Commun. 7, 12842 (2016).

36. Vinnik, D. et al. Magnetic and structural properties of barium hexaferrite $\mathrm{BaFe}_{12} \mathrm{O}_{19}$ from various growth techniques. Materials 10, 578 (2017).

37. Landau, L. D. \& Lifshitz, E. M. in Statistical Physics, Vol. 5 p. ii (Elsevier, 1968).

38. Mashiyama, H., Eric Mkam Tchouobiap, S. \& Ashida, M. Order-disorder and displacive transitions in a quantum Ising model. J. Phys. Soc. Jpn. 77, 084709 (2008).

39. Matsumoto, H. et al. Optical conductivity from local anharmonic phonons. Phys. Rev. B 79, 214306 (2009).

40. Mori, T. et al. Optical conductivity of rattling phonons in type-l clathrate $\mathrm{Ba}_{8} \mathrm{G}_{16} \mathrm{Ge}_{30}$. Phys. Rev. B 79, 212301 (2009).

41. Onodera, Y. Dynamic susceptibility of classical anharmonic oscillator. Prog. Theor. Phys. 44, 1477-1499 (1970).

42. Hlinka, J. et al. Origin of the "waterfall" effect in phonon dispersion of relaxor perovskites. Phys. Rev. Lett. 91, 107602 (2003).

43. Delaire, O. et al. Giant anharmonic phonon scattering in PbTe. Nat. Mater. 10, 614-619 (2011).

44. Silva Júnior, F. M. \& Paschoal, C. W. A. Spin-phonon coupling in $\mathrm{BaFe}_{12} \mathrm{O}_{19}$ M-type hexaferrite. J. Appl. Phys. 116, 244110 (2014).

45. Chen, X.-B. et al. Raman studies of spin-phonon coupling in hexagonal BaFe $_{12} \mathrm{O}_{19}$. J. Appl. Phys. 114, 013912 (2013).

46. Izadkhah, H., Zare, S., Somu, S. \& Vittoria, C. Effects of cobalt substitutions on the magnetoelectric coupling of M-type hexaferrite films. Appl. Phys. Lett. 106, 142905 (2015)

47. Ebnabbasi, K, Mohebbi, M. \& Vittoria, C. Room temperature magnetoelectric effects in bulk poly-crystalline materials of $\mathrm{M}$ - and Z-type hexaferrites. J. Appl. Phys. 113, 17C703 (2013).

48. Mohebbi, M., Ebnabbasi, K. \& Vittoria, C. First observation of magnetoelectric effect in M-type hexaferrite thin films. J. Appl. Phys. 113, 17 C710 (2013).

49. Kumar, K. \& Pandey, D. Quantum phase transitions in $\mathrm{Ba}_{(1-x)} \mathrm{Ca}_{x} \mathrm{Fe}_{12} \mathrm{O}_{19}$

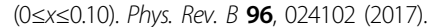

50. Rowley, S. E. et al. Uniaxial ferroelectric quantum criticality in multiferroic hexaferrites $\mathrm{BaFe}_{12} \mathrm{O}_{11}$ and $\mathrm{SrFe}_{12} \mathrm{O}_{10}$. Sci. Rep. 6, 25724 (2016).

51. Rowley, S. E. et al. Ferroelectric quantum criticality. Nat. Phys. 10, 367-372 (2014).

52. Khmel'nitskii, D. E. \& Shneerson, V. L. Russian version. Sov. Phys. Solid State 13, 687 (1971).

53. Khmel'nitskii, D. E. \& Shneerson, V. L. Phase transitions of the displacement type in crystals at very low temperatures. Sov. J. Exp. Theor. Phys. 37, 164 (1973).

54. Lunkenheimer, P. et al. Colossal dielectric constants in transition-metal oxides. Eur. Phys. J. Spec. Top. 180, 61-89 (2009).

55. Pyatakov, A. P. et al. Magnetically switched electric polarity of domain walls in iron garnet films. EPL 93, 17001 (2011).

56. Popov, M. A., Zavislyak, I. V. \& Srinivasan, G. Current tunable barium hexaferrite millimeter wave resonator. Microw. Opt. Technol. Lett. 60, 458-462 (2018).
57. Bierlich, S., Gellersen, F., Jacob, A. \& Töpfer, J. Low-temperature sintering and magnetic properties of Sc- and In-substituted M-type hexagonal barium ferrites for microwave applications. Mater. Res. Bull. 86, 19-23 (2017).

58. Shi, J. et al. THz photonics in two dimensional materials and metamaterials: properties, devices and prospects. J. Mater.Chem C 6, 1291-1306 (2018).

59. $L \mathrm{~V}, \mathrm{~T}$. T. et al. Hybrid metamaterial switching for manipulating chirality based on $\mathrm{VO}_{2}$ phase transition. Sci. Rep. 6, 23186 (2016).

60. Harris, V. G. et al. Ba-hexaferrite films for next generation microwave devices (invited). J. Appl. Phys. 99, 08 M911 (2006).

61. Bowrothu, R., Kim, H.-I. I., Smith, C. S., Arnold, D. P. \& Yoon, Y.-K. K. 35$\mathrm{GHz}$ barium hexaferrite/PDMS composite-based millimeter-wave circulators for $5 \mathrm{G}$ applications. IEEE Trans. Microw. Theory Tech. 68, 5065-5071 (2020).

62. Zhang, G. \& Jiang, Y. A novel CMOS hexaferrite circulator with $25 \mathrm{GHz}$ operating frequency. AIP Adv. 11, 045022 (2021).

63. Sai, R., Shivashankar, S. A., Yamaguchi, M. \& Bhat, N. Magnetic nanoferrites for RF CMOS: enabling $5 \mathrm{G}$ and beyond. Electrochem. Soc. Interface 26, 71 (2017)

64. Srinivasan, G., Popov, M. A. \& Zavislyak, I. V. Chapter 10. Dielectric resonance in ferrites for sub-thz signal-processing devices. Solid State Phys 64, 349-363 (2013).

65. Vendik, I. B. et al. Tunable metamaterials for controlling THz radiation. In IEEE Transactions on Terahertz Science and Technology 538-549 (IEEE, 2012).

66. Popov, M. A., Zavislyak, I. V., Movchan, N. N., Gudim, I. A. \& Srinivasan, G. Mode splitting in $37-42 \mathrm{GHz}$ barium hexaferrite resonator: theory and device applications. IEEE Trans. Magn. 50, 4004507 (2014).

67. Wan, P. et al. Flexible transparent films based on nanocomposite networks of polyaniline and carbon nanotubes for high-performance gas sensing. Small 11, 5409-5415 (2015)

68. Liu, J. et al. MXene-enabled electrochemical microfluidic biosensor: applications toward multicomponent continuous monitoring in whole blood. Adv. Funct. Mater. 29, 1807326 (2019).

69. Yang, S. et al. High sensitivity and good selectivity of ultralong $\mathrm{MoO}_{3}$ nanobelts for trimethylamine gas. Sensors Actuators B Chem 226, 478-485 (2016).

70. Ziegler, D., Marchisio, A., Montanaro, L., Palmero, P. \& Tulliani, J. M. Barium hexaferrite thick-films for ozone detection at low temperature. Solid State lonics 320, 24-32 (2018).

71. Das, T. et al. Novel barium hexaferrite based highly selective and stable trace ammonia sensor for detection of renal disease by exhaled breath analysis. Sensors Actuators B Chem. 325, 128765 (2020).

72. Karmakar, M., Mondal, B., Pal, M. \& Mukherjee, K. Acetone and ethanol sensing of barium hexaferrite particles: a case study considering the possibilities of non-conventional hexaferrite sensor. Sensors Actuators B Chem 190, 627-633 (2014).

73. Antonio-Lopez, J. E., Castillo-Guzman, A., May-Arrioja, D. A., Selvas-Aguilar, R. \& LiKamWa, P. Tunable multimode-interference bandpass fiber filter. Opt. Lett. 35, 324 (2010).

74. Uher, J. \& Hoefer, W. J. R. Tunable microwave and millimeter-wave band-pass filters. IEEE Trans. Microw. Theory Tech. 39, 643-653 (1991).

75. Tan, G. \& Chen, X. Structure and multiferroic properties of barium hexaferrite ceramics. J. Magn. Magn. Mater. 327, 87-90 (2013).

76. Trukhanov, A. V. et al. Multiferroic properties and structural features of M-type Al-substituted barium hexaferrites. Phys. Solid State 59, 737-745 (2017).

77. Karpov, D. et al. Nanoscale topological defects and improper ferroelectric domains in multiferroic barium hexaferrite nanocrystals. Phys. Rev. B 100, 054432 (2019).

78. Born, M. \& Wolf, E. Principles of Optics (Cambridge University Press, 1999).

79. Böhmer, R., Maglione, M., Lunkenheimer, P. \& Loidl, A. Radio-frequency dielectric measurements at temperatures from 10 to $450 \mathrm{~K}$. J. Appl. Phys. 65, 901-904 (1989).

80. Gorshunov, B. et al. Terahertz BWO-spectrosopy. Int. J. Infrared Millim. Waves 26, 1217-1240 (2005)

81. Alyabyeva, L. et al. Bi-relaxor behavior and $\mathrm{Fe}^{2+}$ fine structure in single crystalline Ba0.3Pb0.7Fe12O19M-type hexaferrite. In 2018 43rd International Conference on Infrared, Millimeter, and Terahertz Waves (IRMMW-THz) 18227420 (IEEE, 2018) 